Relations industrielles

Industrial Relations

\title{
Silvana SCIARRA : Contratto collettivo e contrattazione in azienda. Milano, Franco Angeli, 1985, 226 pp.
}

\section{Dimitri Weiss}

Volume 41, numéro 1, 1986

URI : https://id.erudit.org/iderudit/050195ar

DOI : https://doi.org/10.7202/050195ar

Aller au sommaire du numéro

Éditeur(s)

Département des relations industrielles de l'Université Laval

ISSN

0034-379X (imprimé)

1703-8138 (numérique)

Découvrir la revue

Citer ce compte rendu

Weiss, D. (1986). Compte rendu de [Silvana SCIARRA : Contratto collettivo e contrattazione in azienda. Milano, Franco Angeli, 1985, 226 pp.] Relations industrielles / Industrial Relations, 41(1), 198-200.

https://doi.org/10.7202/050195ar

Tous droits réservés (C Département des relations industrielles de l'Université Laval, 1986
Ce document est protégé par la loi sur le droit d'auteur. L'utilisation des services d'Érudit (y compris la reproduction) est assujettie à sa politique d'utilisation que vous pouvez consulter en ligne.

https://apropos.erudit.org/fr/usagers/politique-dutilisation/ 
netteté de la rédaction...), mais aussi cette faille majeure, d'un point de vue scientifique: l'absence d'analyse juridique en fonction de l'arrière-plan de droit civil, qui, pourtant, jouxte la Common Law au pays.

Quant à la mise à jour elle-même, elle ne permettait, au moment où on la rédigeait, que de soulever quelques grandes interrogations quant à l'incidence de la Charte canadienne des droits et libertés sur certains aspects de la législation et des pratiques du travail (clauses de sécurité syndicales, du droit de grève, du piquetage et du boycottage...), sans qu'il puisse encore être fait état de solutions jurisprudentielles significatives à leur sujet. Dans l'ensemble, les autres ajouts se bornent aux décisions les plus significatives des cours et des organismes des provinces de Common Law et à celles du Conseil canadien des relations du travail, à l'exclusion de la jurisprudence québécoise, si ce n'est quelques arrêts de la Cour suprême du Canada.

L'obligation de négocier de bonne foi donne lieu à des passages intéressants sur le contenu des propositions avancées par les parties. Par contre, on peut regretter l'absence de mention du caractère innovateur, à bien des égards, de la Loi sur la santé et la sécurité du travail du Québec, à l'exception de ce qui a trait au refus du travail dangereux. De même en est-il de l'obligation légale de justifier suffisamment le congédiement selon les lois de la NouvelleÉcosse et du Québec, sous réserve de leurs conditions d'application, de l'obligation du vote de grève et du droit du salarié de participer à l'arbitrage des griefs qui le concerne, selon le Code québécois.

L'ensemble, malgré son caractère perfectible, constitue toujours une synthèse fort bien réussie du droit du travail canadien actuel.

Pierre VERGE

\section{Université Laval}

Contratto collettivo e contrattazione in azienda, par Silvana Sciarra, Milano, Franco Angeli, 1985, $226 \mathrm{pp}$.

Cet ouvrage de droit du travail sur «Contrat collectif et négociation dans l'entreprise» ne peut laisser indifférent le lecteur français, et ceci à plus d'un titre. Sa parution coincida, en effet, avec la centralité, en France, du thème de la négociation collective d'entreprise - parallèle à celui de l'entreprise dans la société - non seulement parmi les praticiens et les acteurs du système de relations industrielles, voire ses observateurs patentés, mais également dans le débat politique, où discours, programmes, plate-formes, meetings, tables rondes et autres face-àface, ajoutés au débat parlementaire sur l'aménagement du temps de travail, témoignèrent de l'interêt que lui portèrent les compétiteurs.

Thème indissolublement lié à celui que les Italiens appellent la dérogabilité - posé, d'ailleurs, dans toute son étendue en Italie depuis plusieurs années déjà -, la norme dérogatoire d'essence contractuelle devant remplacer la norme légale ou réglementaire et transformer la négociation collective d'une négociation d'addition, selon le principe du «toujours plus», en une négociation d'acquis de substitution, selon la logique du «donnant donnant». Et ce dans les conditions, délibérées, dans lesquelles les matières faisant l'objet d'une obligation annuelle de négocier sont aussi celles qui permettent la dérogation.

De la, pareillement, les controverses autour du renversement de la hiérarchie des sources de droit et sur la configuration respective de l'ordre public absolu, socle intangible des garanties sociales fondamentales, et de l'ordre public relatif, susceptible de dérogation, dont on n'a pas fini de parler. 
Le problème de l'adaptation des normes de droit à la réalité compétitive de nos jours, qui trouve des traductions spécifiquement hexagonales, se retrouve ailleurs, et notamment de l'autre côté des Alpes. Au début de 1985, Gino Giugni, professeur de droit du travail à l'Université de Rome et président de la commission des lois du Sénat italien, co-auteur de la loi de mai 1970 sur l'exercice des droits syndicaux, dite Statut des travailleurs, suggérait, dans une interview accordée d̀ la revue de la CGT italienne, Rassegna sindacale, de mettre sur pied un système de règles et de garanties flexibles - qui aillent non pas à l'encontre, mais au-delà dudit «statut» - en mesure de supprimer toutes les rigidités actuelles du marché du travail (entrée, conditions de travail, sortie), et d'étendre le domaine des normes «semi-impératives» des contrats collectifs, c'est-à-dire celles qui peuvent être modifiées également «en moins», in peius.

Nous aurons, en substance, ajoutait Giugni, un système disposant d'une série de soupapes d'élasticité, un contrat collectif qui puisse déroger in peius sur des points particuliers, par exemple concessions salariales en échange d'une réduction de la durée du travail, et un contrat de travail qui, en partant des normes de caractère supérieur, pourra définir des conditions différenciées et personnalisées. "La dynamique contractuelle serait énormément freinée s'il était interdit aux syndicats de travailleurs de faire des concessions sur certains sujets de négociation en échange d'améliorations sur d'autres points, considérés à ce moment d'une importance majeure ou, de toute façon, en vue d'objectifs de politique syndicale comme l'emploi, etc.» (Giugni, «Diritto sindacale», Bari, Cacucci editore, $7^{\mathrm{e}}$ éd. 1984, p. 179).

La référence à Giugni n'est, certes, pas fortuite: il fut lè créateur de ce que j'avais appelé l'«École de Bari» en droit du travail, dont Silvana Sciarra fit partie, et il continue aujourd'hui d'animer un pan des plus dynamiques de la doctrine «travailliste» italienne.

Silvana Sciarra, professeur associé à l'Université de Sienne, ne passe pas, naturellement, sous silence la dérogabilité. Elle en parle aussi bien dans le corps de son texte que dans des notes, très utiles, notamment, pour l'orientation bibliographique qu'elles recèlent. Et elle évoque, également, très pertinemment, le processus de «décodification», qui atteint le droit du travail, de même que ce qu'on appelle en Italie la négociation «récessive», c'est-à-dire la négociation de concessions, qui est, dit-elle, toujours négociation collective.

Plus que jamais, pourraient ajouter certains des promoteurs français du projet actuel de «contrat collectif d'entreprise» qui, en liant concession et dérogation, estiment qu'il s'agit de la seule négociation véritablement contractuelle, puisque c'est la dérogation qui donne vie à la notion de contrepartie et que l'accord qui additionne chaque année n'a pas le caractère de contrat.

Silvana Sciarra se réfère, elle, par exemple, à propos des contrats de solidarité italiens, au décret-loi du 4 février 1976, lequel «fait explicitement référence au 'contrat collectif d'entreprise' (p. 133), «instrument par le biais duquel les parties défendent leurs concessions réciproques» (p. 135), «reconnaissant ainsi l'importance d'un accord formalisé autour duquel se soit créé le consensus des travailleurs de l'unité productive» (p. 133). (Ce qui, dans une lecture française déformée, conduirait à lier la négociation d'entreprise au projet d'entreprise, parfaitement absent de l'autre côté des Alpes.) Cette prévision semble importante si on considère que, fut-ce en échange du maintien de l'emploi, un tel accord comporte une réduction de salaire et doit, par conséquent, s'identifier à une source sûre de réglementation des rapports de travail (p. 133).

Il n'existe pas, en Italie, d'obligation de négocier, et S. Sciarra, qui est aussi une comparatiste avertie, le note très bien en confrontant la situation italienne à celle d'autres pays, dont, en particulier, la France. Elle dit, ainsi, en s'y référant, combien cette obligation au niveau de l'entreprise lui semble revêtir une connotation fortement garantiste (p. 146), de réglementation vraie et propre de la procédure contractuelle, en reproposant après la loi Wagner de 1935 et 
d'autres dispositions législatives européennes plus récentes, un modèle d'intervention étatique en matière de négociation collective. Dans la solution italienne, ajoute-t-elle (p. 147), le rôle de la loi est plus circonscrit, de manière à ne pas laisser place à une vision d'institutionnalisation de cette procédure: l'obligation de négocier n'a pas de raison d'apparaître comme le pivot d'un système de rapports qui trouve, justement, le fondement de sa légitimité dans la liberté d'accéder à la confrontation des parties.

La liberté de négocier collectivement, expliquait Sciarra dès le début de son livre (pp. 13-14), peut être insérée, elle aussi, parmi les valeurs constitutionnelles, en tant que manifestation des plus marquantes de la liberté syndicale. Et, alors, l'inclusion de la négociation collective dans la sphère des valeurs fondamentales doit être entendue comme excluant l'intervention de la loi du domaine de l'autonomie privée collective (thèse qui, faut-il ajouter, est remise aujourd'hui de nouveau en question, revitalisée par la Commission parlementaire des réformes institutionnelles qui a proposé, notamment, une modification de l'article 39 de la constitution).

Le livre de S. Sciarra trace, à partir de là, un panorama attentif et critique de la négociation collective dans l'Italie de l'après-guerre, avec la prépondérance et, ensuite, le tassement de la négociation de branche et son ouverture à d'autres niveaux, le rôle contractuel des défuntes commissions internes - à propos desquelles l'auteur traite des notions de communauté d'entreprise et de communauté de travail, aujourd'hui à la mode en France - , la loi de mai 1970 et l'identité des agents contractuels (représentation syndicale d'entreprise ou conseils d'usine?)...

Elle note le rôle "paralégislatif» du contrat collectif, qualifié par la doctrine comme étant «une source de droit agissant sur un plan de parité avec la norme légale» (p. 85), de la période de crise, avec la "Cassa integrazione guadagni», et beaucoup d'autres choses que l'économie de ce compte-rendu empêche d'évoquer.

Son apport, nécessaire et profitable, pédagogiquement bien conçu, m'apparaît également utile au lecteur non eurolatin, y compris nord-américain, qui aura, ainsi, une image exemplaire du cheminement d'un processus qui a parcouru, pour s'instaurer, un itinéraire sinueux mais, néanmoins, digne d'attention et de réflexion. Un bon livre.

Dimitri WEISS

Université de Paris I Panthéon-Sorbonne

Le conflit du travail: stratégie et tactique, par Gilles Plante, Coll. Relations du Travail, Département des relations industrielles, Les Presses de l'Université Laval, Québec, 1984, 164 pp., ISBN 2-7637-7041-X

Ce livre est précis, clair et intelligent mais il l'est dans des limites trop étroites pour que le modèle qu'il nous propose des règlements de conflits en période de négociation (ou renégociation) de conventions collectives entre patrons et syndicats soit adéquat à la complexité des rapports sociaux en jeu. Nous aurons à rendre crédible cette critique de fond d'un livre lu très attentivement.

L'essai de Gilles Plante est fait d'une suite de brefs chapitres, les six premiers proposent le modele, les deux derniers illustrent son application à deux cas. Deux annexes présentent des documents pertinents à ces deux cas. Quatre-vingt-deux pages sur le modèle, autant sur les deux cas avec les annexes.

En suivant de près l'introduction de l'auteur (p. 1-3), voici à quoi ressemble le contenu du livre. Au Chapitre I (p. 4-14), nous «allons apprendre que l'esprit de la guerre anime les relations entre employeurs et associations de salariés» (p. 1). Le Chapitre II s'intéresse aux «res- 